\title{
Scope of Intelligence Approcahes for Unit Commitment Under Uncertain Sustainable Energy Environment For Effective Vehicle To Grid Operations-A Comprehensive Review
}

\author{
Dinesh Dhawale* ${ }^{1,2}$, and Vikram Kumar Kamboj ${ }^{1}$ \\ ${ }^{1}$ School of Electronics and Electrical Engineering, Lovely Professional University, Punjab, INDIA \\ ${ }^{2}$ Department of Electrical Engineering, Priyadarshini College of Engineering, Nagpur, Maharashtra, INDIA
}

\begin{abstract}
Electric vehicles are getting popularity as these are eco-friendly and could be a part of power sector in the future. Electric Vehicles are the smart hybrid vehicles, which stores electric power during their operation, which could be stored in storage cells. These electric vehicles may be plug-in electric vehicles or battery operated electric vehicles. The concept of aggregators may be utilized, wherein the stored energy in vehicles could be supplied to grid during parking hours .This also facilitate the consumers to sale power during the high power demand and purchase power during low power demand. Thus, a bi-directional flow of power could be possible either from vehicle to grid or vice-versa. A large penetration of electric vehicles could result in increase in power demand which could be compensated by proper coordinated unit commitment and optimization techniques. The increasing load on grid by the impact of demand and trends in small generating units which require proper selection of number of generating units to put in line and other units in off condition calls for the concept of unit commitment. It is the selection of more efficient units to be in service and shutting down the other unit while maintaining all the other constraint constant. This would result in effective power flow in an economic manner, simultaneously maintaining the adequacy and reliability of the system. The proposed research represents the scope of intelligence algorithm for unit commitment problem with effective solution of vehicle to grid operations along with sustainable energy for realistic power system.
\end{abstract}

\section{INTRODUCTION}

In the era of development and advancement in technology, electrical power plays a vital role as power is essential commodity for the functioning of any industrial growth. As the main source of electrical energy are fossil fuel energy sources which are diminishing rapidly and would lead to an end one day. This requires careful attention to make systematically and economically the use of these resources. Also ,the power generation companies needs to motivate the small power industries to motivate the small power generation units to take part in the contribution to take part in the contribution to decrease the threads of power crisis. As a necessity one should make enough attempts to utilize non-conventional energy sources so that the burden on conventional energy source could be reduced to a larger extend. This would result in more and more involvement of sustainable energy sources. But as we know that these sources are intermittent energy sources, as they do not provide a constant output power. Transforming present energy system towards one emphasized by renewable energy comes with some challenges, leads to make proper co-ordinate use of conventional and sustainable energy sources provides the optimum power generation and transmission could be possible maintaining all the constraints constant. Secondly, the invent of hybrid electric vehicles could also play an important role to reduce the dependency on conventional energy source. Thus, the proposed research proposal is the development of a hybrid system which combines the overall features of unit

\footnotetext{
* Corresponding author: $\underline{\text { ddhawale56@gmail.com }}$
} 
commitment, renewable energy sources with the effect of hybrid electric vehicles.

\section{LITERATURE REVIEW}

Electrical power is generated by various power generating units such as thermal, hydro, nuclear, solar and wind etc. These generating units are required to turn on and off in proper sequence, while doing so the expense of on off/ should be minimum. This process of analysis and continuously changing generating schedule is referred to as unit commitment (UC)[1].Unit commitment is an optimization problem used to select the operation schedule at each hour interval with changing loads within permissible limit. Also due to large penetration of intermittent sources led to more complex in satisfying the supply and demand. Esmaeeli et al.[2] in his work suggested that with increase in wind power, power generation flexibility must increase as well. Jonghe et al. [3] describes the various complexities introduced in generation schedule by these intermittent sources. Shahriar et al. [4] presents a new technique to solve problems due to renewable energy penetration by formulating a Mixed Integer Linear Programming (MILP) technique. Further, Wang et al. [5] presents the increased complexity due to introduction of large electric vehicles as new participants in the existing power system. However, Vehicle to grid (V2G) can help to improve the reliability and stability of the grid, alleviate power shortages, and reduce peak power, spinning reserve, voltage and frequency regulation .Yang et al. [6] proposed a hybrid meta-heuristic method for solving mixed integer unit commitment problem integrating with significant plug-in electric vehicles. These electric vehicles can act as storage cells when large number of batteries are connected to form aggregators. These aggregators may be used as spinning reserve thus reducing dependency on conventional generating sources. The combined activity of the hybrid power system should be utilized appreciably in intellectual manner to effectively produce economic generation schedule. This calls up for new novel algorithms for providing more optimal solutions to these hybrid UC problems .Dynamic programming (DP)[7], Lagrangian relaxation(LR)[8],Harmony search (HS)[9], Particle swarm optimization (PSO)[10], Genetic algorithm(GA)[11] are some of the recent optimization methods to handle conventional UC problem. Seth et al. [12] in his work describes improved priority list method to effectively a ramp rate constrained unit commitment.Table-1 presents a comprehensive review of some related intelligent and innovative approach to tackle complex UC problems.

Table-1: Comprehensive review of UC papers

\begin{tabular}{|c|c|c|c|c|}
\hline Author & $\begin{array}{l}\text { Year of } \\
\text { Publicati } \\
\text { on }\end{array}$ & $\begin{array}{l}\text { Refer } \\
\text { ences }\end{array}$ & Brief Review & Constraints \\
\hline $\begin{array}{l}\text { A. Y. } \\
\text { Saber } \\
\text { and G. } \\
\text { K. }\end{array}$ & 2009 & {$[10]$} & $\begin{array}{l}\text { This paper } \\
\text { presents a } \\
\text { discrete particle } \\
\text { swarm }\end{array}$ & $\begin{array}{l}\text { Power } \\
\text { balance, } \\
\text { V2G, } \\
\text { Spinning }\end{array}$ \\
\hline
\end{tabular}

\begin{tabular}{|c|c|c|c|c|}
\hline $\begin{array}{l}\text { Venaya } \\
\text { gamoort } \\
\text { hy }\end{array}$ & & & $\begin{array}{l}\text { optimization } \\
\text { technique used } \\
\text { to optimize the } \\
\text { scheduling of } \\
\text { gridable } \\
\text { vehicles. PSO } \\
\text { method used } \\
\text { also reduces } \\
\text { operation cost } \\
\text { by charging the } \\
\text { vehicles from } \\
\text { renewable } \\
\text { sources or utility } \\
\text { during off-peak } \\
\text { load when price } \\
\text { is low and } \\
\text { discharge to grid } \\
\text { at peak load } \\
\text { when price is } \\
\text { high (V2G) }\end{array}$ & $\begin{array}{l}\text { reserve, } \\
\text { Generation } \\
\text { limits, } \\
\text { Vehicle } \\
\text { parking } \\
\text { limits, } \\
\text { Transmissio } \\
\text { n constraints }\end{array}$ \\
\hline $\begin{array}{l}\text { M. } \\
\text { Singh, } \\
\text { I. Kar, } \\
\text { and P. } \\
\text { Kumar }\end{array}$ & 2010 & [13] & $\begin{array}{l}\text { This paper } \\
\text { presents } \\
\text { simulation of } \\
\text { actual data of } \\
\text { Guwahati city } \\
\text { with due effect } \\
\text { of EV } \\
\text { penetration .It is } \\
\text { observed that } \\
\text { coordinated } \\
\text { charging and } \\
\text { discharging } \\
\text { patterns not only } \\
\text { flatten the } \\
\text { voltage profile } \\
\text { but also reduces } \\
\text { the transmission } \\
\text { losses }\end{array}$ & $\begin{array}{l}\text { Slow } \\
\text { Charging, } \\
\text { Medium } \\
\text { Charging, } \\
\text { Fast } \\
\text { Charging, } \\
\text { EV } \\
\text { Coordinated }\end{array}$ \\
\hline $\begin{array}{l}\text { B. } \\
\text { Palminti } \\
\text { er and } \\
\text { M. } \\
\text { Webster }\end{array}$ & 2011 & [14] & $\begin{array}{l}\text { In this research, } \\
\text { unit } \\
\text { commitment } \\
\text { problem is } \\
\text { combined with } \\
\text { into a single } \\
\text { mixed-integer } \\
\text { optimization } \\
\text { which focuses } \\
\text { on the dynamic } \\
\text { thermal power } \\
\text { system. Also } \\
\text { suggested that } \\
\text { for achieving } \\
\text { carbon emission } \\
\text { targets policy } \\
\text { makers may } \\
\text { approach by } \\
\text { providing } \\
\text { incentives to } \\
\text { customers to } \\
\text { gain regulatory } \\
\text { goals }\end{array}$ & $\begin{array}{l}\text { Economic } \\
\text { Dispatch, } \\
\text { Generation } \\
\text { Expansion, } \\
\text { Expansion } \\
\text { planning, } \\
\text { Operating } \\
\text { Reserve }\end{array}$ \\
\hline $\begin{array}{l}\text { C. De } \\
\text { Jonghe, } \\
\text { E. } \\
\text { Delarue } \\
\text {, R. } \\
\text { Belman } \\
\text { s, and }\end{array}$ & 2011 & [3] & $\begin{array}{l}\text { In this research, } \\
\text { an alternative } \\
\text { methodology is } \\
\text { introduced } \\
\text { which provides } \\
\text { system } \\
\text { flexibility with }\end{array}$ & $\begin{array}{l}\text { Operational } \\
\text { Constriants, } \\
\text { Must run } \\
\text { level, Ramp } \\
\text { rate } \\
\text { constraints, } \\
\text { Maintenance }\end{array}$ \\
\hline
\end{tabular}




\begin{tabular}{|c|c|c|c|c|}
\hline $\begin{array}{l}\text { W. } \\
\text { D'haese } \\
\text { leer }\end{array}$ & & & $\begin{array}{l}\text { better storage } \\
\text { capacity. It also } \\
\text { provides } \\
\text { analysis of the } \\
\text { impact of a high } \\
\text { level wind } \\
\text { penetration on } \\
\text { the optimal } \\
\text { generation by } \\
\text { combining } \\
\text { screening curve } \\
\text { methodology } \\
\text { with a linear } \\
\text { programming } \\
\text { (LP) }\end{array}$ & Constraints \\
\hline $\begin{array}{l}\text { S. } \\
\text { Kamboj } \\
\text {, W. } \\
\text { Kempto } \\
\text { n, and } \\
\text { K. S. } \\
\text { Decker }\end{array}$ & 2011 & [15] & $\begin{array}{l}\text { This paper } \\
\text { presents a } \\
\text { deployed multi- } \\
\text { agent system } \\
\text { which integrate } \\
\text { electric drive } \\
\text { vehicles to grid. } \\
\text { These power } \\
\text { regulations } \\
\text { markets depends } \\
\text { upon ancillary } \\
\text { (eg. Spinning } \\
\text { reserve) and } \\
\text { non-ancillary } \\
\text { (eg. peak power) } \\
\text { services. A } \\
\text { detailed study of } \\
\text { V2G, system } \\
\text { equipment's and } \\
\text { associated } \\
\text { accessories for } \\
\text { power flow } \\
\text { within the } \\
\text { standard } \\
\text { constraint. }\end{array}$ & $\begin{array}{l}\text { Maximum } \\
\text { rate of } \\
\text { charging, } \\
\text { Maximum } \\
\text { regulation- } \\
\text { up power, } \\
\text { Maximum } \\
\text { regulation- } \\
\text { down power }\end{array}$ \\
\hline $\begin{array}{l}\text { G. O. } \\
\text { Suvire, } \\
\text { M. G. } \\
\text { Molina, } \\
\text { and P. } \\
\text { E. } \\
\text { Mercad } \\
\text { o }\end{array}$ & 2012 & [16] & $\begin{array}{l}\text { In this research, } \\
\text { dynamic model } \\
\text { is developed } \\
\text { which stores } \\
\text { energy produced } \\
\text { due to wind } \\
\text { generation and } \\
\text { integrated with } \\
\text { microgrids. This } \\
\text { hybrid system } \\
\text { controls voltage, } \\
\text { frequency and } \\
\text { smooth's active } \\
\text { fluctuations due } \\
\text { to DG } \\
\text { penetration. }\end{array}$ & $\begin{array}{l}\text { Dc link } \\
\text { voltage, } \\
\text { variable } \\
\text { speed, } \\
\text { Sudden } \\
\text { change in } \\
\text { load }\end{array}$ \\
\hline $\begin{array}{l}\text { A. } \\
\text { Foley, } \\
\text { B. } \\
\text { Tyther, } \\
\text { P. } \\
\text { Calnan, } \\
\text { and B. } \\
\text { Ó } \\
\text { Gallach } \\
\text { óir }\end{array}$ & 2013 & [17] & $\begin{array}{l}\text { In this research, } \\
\text { of EV charging } \\
\text { in the single } \\
\text { whole electricity } \\
\text { market in } \\
\text { Ireland is } \\
\text { analyzed. EV } \\
\text { charging under } \\
\text { peak and off- } \\
\text { peak charging } \\
\text { scenario's is } \\
\text { examined. It is }\end{array}$ & $\begin{array}{l}\text { Ramp rate } \\
\text { up and } \\
\text { down, } \\
\text { Minimum up } \\
\text { and down } \\
\text { time, } \\
\text { Emission } \\
\text { coefficients }\end{array}$ \\
\hline
\end{tabular}

\begin{tabular}{|c|c|c|c|c|}
\hline & & & $\begin{array}{l}\text { seen that } \\
\text { introduction of } \\
\text { V2G technology } \\
\text { increases power } \\
\text { system } \\
\text { flexibility and } \\
\text { facilitates more } \\
\text { penetration of } \\
\text { wind power }\end{array}$ & \\
\hline $\begin{array}{l}\text { Provas } \\
\text { Kumar } \\
\text { Roy }\end{array}$ & 2013 & {$[18]$} & $\begin{array}{l}\text { In this research, } \\
\text { behavior of all } \\
\text { the masses in } \\
\text { the universe due } \\
\text { to gravitational } \\
\text { forces logic is } \\
\text { applied to } \\
\text { thermal unit } \\
\text { commitment } \\
\text { problem. The } \\
\text { proposed } \\
\text { algorithm is } \\
\text { tested using } \\
\text { Matlab } \\
\text { programming } \\
\text { and results are } \\
\text { compared with } \\
\text { other known } \\
\text { algorithms for } \\
\text { six systems } \\
\text { during a } \\
\text { schedule of } \\
24 \mathrm{H} \text {. }\end{array}$ & $\begin{array}{l}\text { unit capacity } \\
\text { limit, ramp } \\
\text { rate limits, } \\
\text { spinning } \\
\text { reserve con- } \\
\text { straints, } \\
\text { minimum up } \\
\text { time and } \\
\text { down time } \\
\text { constraints }\end{array}$ \\
\hline $\begin{array}{l}\text { L. Liu, } \\
\text { H. Li, } \\
\text { Y. Xue, } \\
\text { and W. } \\
\text { Liu }\end{array}$ & 2014 & [19] & $\begin{array}{l}\text { In this research, } \\
\text { reactive power } \\
\text { is compensated } \\
\text { using } \\
\text { compensation } \\
\text { algorithm which } \\
\text { tackles system } \\
\text { operation issues } \\
\text { with improved } \\
\text { system stability } \\
\text { and reliability. } \\
\text { Exact power } \\
\text { distribution is } \\
\text { made by proper } \\
\text { coordination } \\
\text { between power } \\
\text { variables. Thus, } \\
\text { proposed } \\
\text { method } \\
\text { effectively } \\
\text { improves system } \\
\text { performance in } \\
\text { terms of stability } \\
\text { and reliability. }\end{array}$ & $\begin{array}{l}\text { maximum } \\
\text { power point } \\
\text { tracking, } \\
\text { Reactive } \\
\text { power, } \\
\text { Capacitor } \\
\text { charging }\end{array}$ \\
\hline $\begin{array}{l}\text { M. E. } \\
\text { El- } \\
\text { Hawary }\end{array}$ & 2014 & [20] & $\begin{array}{l}\text { This research } \\
\text { presents the } \\
\text { benefits of smart } \\
\text { grid such as } \\
\text { environment and } \\
\text { socio-economic. } \\
\text { It also presents } \\
\text { the dynamic } \\
\text { interactive, real } \\
\text { time } \\
\text { infrastructure } \\
\text { and building the }\end{array}$ & $\begin{array}{l}\text { Charging } \\
\text { and } \\
\text { Discharging } \\
\text { Constraints }\end{array}$ \\
\hline
\end{tabular}




\begin{tabular}{|c|c|c|c|c|}
\hline & & & $\begin{array}{l}\text { power system of } \\
\text { the future } \\
\text { through smart } \\
\text { grid. It also } \\
\text { provides all } \\
\text { information, } \\
\text { possibilities, } \\
\text { polices and } \\
\text { benefits in } \\
\text { designing a } \\
\text { smart intelligent } \\
\text { system }\end{array}$ & \\
\hline $\begin{array}{l}\text { Yaowen } \\
\text { Yu, } \\
\text { Peter B. } \\
\text { Luh, } \\
\text { Eugene } \\
\text { Litvino } \\
\text { v, } \\
\text { Tongxin } \\
\text { Zheng, } \\
\text { Senior, } \\
\text { Jinye } \\
\text { Zhao, } \\
\text { and } \\
\text { Feng } \\
\text { Zhao }\end{array}$ & 2014 & [21] & $\begin{array}{l}\text { This research } \\
\text { presents a } \\
\text { combined } \\
\text { Markovian and } \\
\text { Interval unit } \\
\text { commitment to } \\
\text { solve the } \\
\text { problem of } \\
\text { congestion } \\
\text { created due to } \\
\text { uncertain nature } \\
\text { of wind } \\
\text { generation. In } \\
\text { this method } \\
\text { global node } \\
\text { states are taken } \\
\text { care by interval } \\
\text { optimization and } \\
\text { local nodes } \\
\text { states are } \\
\text { handled by } \\
\text { Markovian } \\
\text { strategy. }\end{array}$ & $\begin{array}{l}\text { Start-up } \\
\text { constraints, } \\
\text { Minimum } \\
\text { up/down, } \\
\text { ramp rate } \\
\text { constraints, } \\
\text { generator } \\
\text { capacity } \\
\text { constraints }\end{array}$ \\
\hline $\begin{array}{l}\text { V.K. } \\
\text { Kamboj } \\
\text {, S. K. } \\
\text { Bath, J. } \\
\text { S. } \\
\text { Dhillon }\end{array}$ & 2015 & {$[22]$} & $\begin{array}{l}\text { In this research, } \\
\text { local search } \\
\text { ability of DE is } \\
\text { improved by } \\
\text { utilizing random } \\
\text { search approach } \\
\text { to enhance } \\
\text { exploitation } \\
\text { phase and is } \\
\text { then applied to } \\
\text { UCP. The } \\
\text { hybrid system is } \\
\text { tested for } \\
\text { standard } \\
\text { benchmark } \\
\text { function and } \\
\text { engineering } \\
\text { problems for } 30 \\
\text { trials and } 500 \\
\text { iterations. }\end{array}$ & $\begin{array}{l}\text { Power } \\
\text { balance } \\
\text { constraints, } \\
\text { Spinning } \\
\text { reserve, } \\
\text { Thermal } \\
\text { constraints }\end{array}$ \\
\hline $\begin{array}{l}\text { C. } \\
\text { CHEN, } \\
\text { S. } \\
\text { DUAN }\end{array}$ & 2015 & {$[23]$} & $\begin{array}{l}\text { In this research, } \\
\text { impact of } \\
\text { different } \\
\text { charging } \\
\text { /discharging } \\
\text { patterns on } \\
\text { microgrids is } \\
\text { analyzed using } \\
\text { genetic } \\
\text { algorithm and } \\
\text { economic } \\
\text { schedule is } \\
\text { evaluated for a }\end{array}$ & $\begin{array}{l}\text { Minimum } / \mathrm{m} \\
\text { aximum } \\
\text { power limits, } \\
\text { Energy } \\
\text { capacity }\end{array}$ \\
\hline
\end{tabular}

\begin{tabular}{|c|c|c|c|c|}
\hline & & & $\begin{array}{l}\text { typical grid- } \\
\text { connected } \\
\text { microgrid. Three } \\
\text { modes of } \\
\text { operation are } \\
\text { presented. A } \\
\text { balance energy } \\
\text { exchange } \\
\text { between PHEVs } \\
\text { and microgrids } \\
\text { is presented with } \\
\text { reduced cost and } \\
\text { improved } \\
\text { efficiency. }\end{array}$ & \\
\hline $\begin{array}{l}\text { H. LIU, } \\
\text { P. } \\
\text { ZENG, } \\
\text { J. GUO, } \\
\text { H. WU, } \\
\text { and S. } \\
\text { GE }\end{array}$ & 2015 & {$[24]$} & $\begin{array}{l}\text { In this research, } \\
\text { controlled EV } \\
\text { charging } \\
\text { strategy with } \\
\text { due } \\
\text { consideration of } \\
\text { sustainable } \\
\text { power outputs is } \\
\text { effectively } \\
\text { employed using } \\
\text { genetic } \\
\text { algorithm. } \\
\text { Furthermore, } \\
\text { considering the } \\
\text { limitations of } \\
\text { sustainable } \\
\text { energy sources, } \\
\text { the developed } \\
\text { hybrid system } \\
\text { provides } \\
\text { improved } \\
\text { economic } \\
\text { scheduling }\end{array}$ & $\begin{array}{l}\text { Charging } \\
\text { and } \\
\text { Discharging } \\
\text { Constraints }\end{array}$ \\
\hline $\begin{array}{l}\text { S. } \\
\text { Umama } \\
\text { heswara } \\
\mathrm{n} \text { and S. } \\
\text { Rajiv }\end{array}$ & 2015 & {$[25]$} & $\begin{array}{l}\text { In this paper, a } \\
\text { review of } \\
\text { benefits and } \\
\text { barriers of } \\
\text { renewable } \\
\text { energy } \\
\text { generation } \\
\text { subjected to } \\
\text { various financial } \\
\text { and social } \\
\text { constraints are } \\
\text { studied. It } \\
\text { further reviews } \\
\text { the emerging } \\
\text { trends and } \\
\text { barriers in the } \\
\text { financing } \\
\text { landscape and } \\
\text { analyses the } \\
\text { impact of policy } \\
\text { performance in } \\
\text { this context. }\end{array}$ & $\begin{array}{l}\text { Wind and } \\
\text { solar } \\
\text { uncertainties }\end{array}$ \\
\hline $\begin{array}{l}\text { N. } \\
\text { Zhang, } \\
\text { Z. Hu, } \\
\text { X. Han, } \\
\text { J. } \\
\text { Zhang, } \\
\text { and Y. }\end{array}$ & 2015 & [26] & $\begin{array}{l}\text { This paper } \\
\text { presents a novel } \\
\text { unit } \\
\text { commitment } \\
\text { model using } \\
\text { fuzzy sequence } \\
\text { which }\end{array}$ & $\begin{array}{l}\text { Power } \\
\text { balance } \\
\text { constraint, } \\
\text { Spinning } \\
\text { reserve } \\
\text { constraint } \\
\text { Minimum }\end{array}$ \\
\hline
\end{tabular}




\begin{tabular}{|c|c|c|c|c|}
\hline Zhou & & & $\begin{array}{l}\text { effectively } \\
\text { modifies } \\
\text { constraints to } \\
\text { eliminate the } \\
\text { hurdles posed } \\
\text { due to uncertain } \\
\text { characteristics } \\
\text { of sustainable } \\
\text { energy sources. } \\
\text { It further } \\
\text { explains how the } \\
\text { power available } \\
\text { in storage cell } \\
\text { act as a reserve } \\
\text { during } \\
\text { unavailability of } \\
\text { wind power } \\
\text { while satisfying } \\
\text { the demand with } \\
\text { low cost and } \\
\text { improved } \\
\text { efficiency. }\end{array}$ & $\begin{array}{l}\text { on/off time } \\
\text { constraint, } \\
\text { Upper limit } \\
\text { of wind } \\
\text { power } \\
\text { penetration } \\
\text { rate }\end{array}$ \\
\hline $\begin{array}{l}\text { K. S. } \\
\text { Reddy, } \\
\text { L. K. } \\
\text { Panwar, } \\
\text { and R. } \\
\text { Kumar }\end{array}$ & 2015 & [27] & $\begin{array}{l}\text { This paper } \\
\text { presents reserve } \\
\text { system in unit } \\
\text { commitment in } \\
\text { deregulated } \\
\text { market by the } \\
\text { participation of } \\
\text { electric vehicles } \\
\text { as an responsive } \\
\text { reserve to } \\
\text { enhance power } \\
\text { system } \\
\text { reliability at } \\
\text { reasonable cost. } \\
\text { It further } \\
\text { encourage to } \\
\text { earn more } \\
\text { revenue from } \\
\text { the required } \\
\text { reserve facility } \\
\text { in the event of } \\
\text { outage or } \\
\text { sudden demand } \\
\text { by satisfying } \\
\text { through electric } \\
\text { vehicle as } \\
\text { reserve. }\end{array}$ & $\begin{array}{l}\text { Load } \\
\text { balance } \\
\text { constraint, } \\
\text { Responsive } \\
\text { reserve } \\
\text { constraint, } \\
\text { Thermal unit } \\
\text { constraints, } \\
\text { Minimum } \\
\text { up/ down } \\
\text { time, Ramp } \\
\text { up/down } \\
\text { rates }\end{array}$ \\
\hline $\begin{array}{l}\text { L. } \\
\text { Yang, J. } \\
\text { Jian, Z. } \\
\text { Dong, } \\
\text { and C. } \\
\text { Tang }\end{array}$ & 2016 & {$[28]$} & $\begin{array}{l}\text { In this research, } \\
\text { proposed } \\
\text { method converts } \\
\text { the conventional } \\
\text { UC problem into } \\
\text { a mixed integer } \\
\text { linear } \\
\text { programming by } \\
\text { combining } \\
\text { several small } \\
\text { non-linear } \\
\text { problems and } \\
\text { provides better } \\
\text { solutions with } \\
\text { less iterations } \\
\text { and also reduced } \\
\text { simulation time } \\
\text { as compared to } \\
\text { original method. }\end{array}$ & $\begin{array}{l}\text { Linear and } \\
\text { non- linear } \\
\text { constraints }\end{array}$ \\
\hline
\end{tabular}

\begin{tabular}{|c|c|c|c|c|}
\hline $\begin{array}{l}\text { W. S. } \\
\text { Tan and } \\
\text { M. } \\
\text { Shaaban }\end{array}$ & 2016 & [29] & $\begin{array}{l}\text { In this research, } \\
\text { MILP } \\
\text { formulation is } \\
\text { used for solving } \\
\text { ramping } \\
\text { capability and } \\
\text { operating } \\
\text { reserve problem } \\
\text { related to } \\
\text { traditional UC } \\
\text { by } \\
\text { implementing a } \\
\text { chance } \\
\text { constraint set. } \\
\text { Further, to } \\
\text { improve } \\
\text { computational } \\
\text { efficiency, } \\
\text { constraint set is } \\
\text { adjusted using } \\
\text { projected } \\
\text { disjunctive } \\
\text { programming. }\end{array}$ & $\begin{array}{l}\text { Probabilistic } \\
\text { chance } \\
\text { constraint }\end{array}$ \\
\hline $\begin{array}{l}\text { V. K. } \\
\text { Kamboj } \\
\text {, S.K. } \\
\text { Bath , } \\
\text { J.S. } \\
\text { Dhillon }\end{array}$ & 2016 & [9] & $\begin{array}{l}\text { In this research, } \\
\text { exploitation } \\
\text { phase is } \\
\text { enhanced by } \\
\text { combining } \\
\text { random search } \\
\text { algorithm for } \\
\text { solving UC } \\
\text { problem. This } \\
\text { hybrid scheme } \\
\text { inspired from } \\
\text { musical tunes is } \\
\text { found to be } \\
\text { divergence free } \\
\text { and able to } \\
\text { handle discrete } \\
\text { and continuous } \\
\text { variables } \\
\text { without prior } \\
\text { setting of } \\
\text { variables. Thus, } \\
\text { it utilizes } \\
\text { various inherent } \\
\text { qualities to } \\
\text { provide } \\
\text { effective } \\
\text { solution for a } \\
\text { particular } \\
\text { optimization } \\
\text { problem }\end{array}$ & $\begin{array}{l}\text { Load } \\
\text { balance } \\
\text { constraint, } \\
\text { Reserve } \\
\text { constraint, } \\
\text { Thermal unit } \\
\text { constraints }\end{array}$ \\
\hline $\begin{array}{l}\text { R. Á. } \\
\text { Fernánd } \\
\text { ez, F. B. } \\
\text { Cillerue } \\
\text { lo, and } \\
\text { I. V. } \\
\text { Martíne } \\
\text { z }\end{array}$ & 2016 & {$[30]$} & $\begin{array}{l}\text { In this research, } \\
\text { extended range } \\
\text { electric vehicle } \\
\text { (EREV) based } \\
\text { in a fuel cell } \\
\text { electric vehicles } \\
\text { (FCEV) set } \\
\text { model is } \\
\text { presented to } \\
\text { achieve better } \\
\text { efficiency and } \\
\text { performance. } \\
\text { This article } \\
\text { shows various } \\
\text { possibilities of }\end{array}$ & $\begin{array}{l}\text { Cut-off } \\
\text { voltage, } \\
\text { Charging } \\
\text { Constraints, } \\
\text { Discharging } \\
\text { constraints }\end{array}$ \\
\hline
\end{tabular}




\begin{tabular}{|c|c|c|c|c|}
\hline & & & $\begin{array}{l}\text { increased run- } \\
\text { range, fuel- } \\
\text { efficiency by the } \\
\text { combined action } \\
\text { of electric } \\
\text { vehicle, } \\
\text { combustion } \\
\text { engine and } \\
\text { hydrogen fuel. } \\
\text { A combined } \\
\text { effect of all } \\
\text { these } \\
\text { participants will } \\
\text { result in better } \\
\text { performance and } \\
\text { accurate energy } \\
\text { management. }\end{array}$ & \\
\hline $\begin{array}{l}\text { K. S. } \\
\text { Reddy, } \\
\text { L. K. } \\
\text { Panwar, } \\
\text { R. } \\
\text { Kumar, } \\
\text { and B. } \\
\text { K. } \\
\text { Panigra } \\
\text { hi }\end{array}$ & 2016 & [31] & $\begin{array}{l}\text { In this paper } \\
\text { fireworks } \\
\text { algorithm is } \\
\text { developed to } \\
\text { improve dual } \\
\text { objective } \\
\text { function } \\
\text { including both } \\
\text { emissions and } \\
\text { cost using } \\
\text { Electric vehicles } \\
\text { and Renewable } \\
\text { energy } \\
\text { technologies. In } \\
\text { this paper author } \\
\text { effectively } \\
\text { utilizes the logic } \\
\text { of sparkles } \\
\text { travel in space } \\
\text { to find the } \\
\text { objective } \\
\text { function }\end{array}$ & $\begin{array}{l}\text { Load } \\
\text { balance } \\
\text { constraint, } \\
\text { Reserve } \\
\text { constraint, } \\
\text { Thermal unit } \\
\text { constraints }\end{array}$ \\
\hline $\begin{array}{l}\text { V. } \\
\text { Monteir } \\
\text { o, J. G. } \\
\text { Pinto, } \\
\text { and J. } \\
\text { L. } \\
\text { Afonso }\end{array}$ & 2016 & [32] & $\begin{array}{l}\text { This research } \\
\text { presents, a } \\
\text { hybrid prototype } \\
\text { battery charger } \\
\text { which operates } \\
\text { in five different } \\
\text { modes which } \\
\text { includes } \\
\text { charging } \\
\text { vehicles and } \\
\text { dispatching } \\
\text { power either } \\
\text { from V2G and } \\
\text { G2V.Also, } \\
\text { based on the } \\
\text { available } \\
\text { research theory, } \\
\text { advantages, } \\
\text { benefits and } \\
\text { control } \\
\text { strategies of } \\
\text { proposed smart } \\
\text { grid model are } \\
\text { elaborately } \\
\text { described. }\end{array}$ & $\begin{array}{l}\text { Charging } \\
\text { and } \\
\text { Discharging } \\
\text { Constraints }\end{array}$ \\
\hline $\begin{array}{l}\text { Z. Bie, } \\
\text { H. Xie, } \\
\text { G. } \mathrm{Hu},\end{array}$ & 2016 & [33] & $\begin{array}{l}\text { In this research, } \\
\text { a novel optimal } \\
\text { scheduling is }\end{array}$ & $\begin{array}{l}\text { Power } \\
\text { balance, } \\
\text { Ramp }\end{array}$ \\
\hline
\end{tabular}

\begin{tabular}{|c|c|c|c|c|}
\hline $\begin{array}{l}\text { and } \mathrm{G} . \\
\mathrm{Li}\end{array}$ & & & $\begin{array}{l}\text { employed using } \\
\text { decoupling } \\
\text { technique to } \\
\text { satisfy demand } \\
\text { response and } \\
\text { improve } \\
\text { economy by } \\
\text { controlling } \\
\text { randomness in } \\
\text { generation } \\
\text {.Further, it } \\
\text { reveals that } \\
\text { operational cost } \\
\text { will vary } \\
\text { according to } \\
\text { customer power } \\
\text { usage and wind } \\
\text { uncertainties. }\end{array}$ & $\begin{array}{l}\text { constraints, } \\
\text { Operation } \\
\text { time } \\
\text { constraints, } \\
\text { Spinning } \\
\text { reserve } \\
\text { constraints, } \\
\text { Customer } \\
\text { satisfaction } \\
\text { constraints, } \\
\text { Demand } \\
\text { power } \\
\text { constraints }\end{array}$ \\
\hline $\begin{array}{l}\text { E.S. Ali } \\
\text {, S.M. } \\
\text { Abd } \\
\text { Elazim, } \\
\text { A.Y. } \\
\text { Abdelaz } \\
\text { iz }\end{array}$ & 2016 & {$[34]$} & $\begin{array}{l}\text { This study } \\
\text { describes that } \\
\text { power } \\
\text { transmission } \\
\text { losses may be } \\
\text { reduced by } \\
\text { using PV system } \\
\text { and wind } \\
\text { generation } \\
\text { through } \\
\text { distributed } \\
\text { generation. In } \\
\text { the proposed ant } \\
\text { lion algorithm } \\
\text { allocation and } \\
\text { sizing of DG } \\
\text { sources } \\
\text { generates } \\
\text { optimum power } \\
\text { with minimum } \\
\text { losses. }\end{array}$ & $\begin{array}{l}\text { Power } \\
\text { conservation } \\
\text { constraint, } \\
\text { Voltage } \\
\text { constraint, } \\
\text { DG limits } \\
\text { constraint, } \\
\text { Line } \\
\text { Capacity } \\
\text { Constraint }\end{array}$ \\
\hline $\begin{array}{l}\text { C. } \\
\text { Deckmy } \\
\text { n, J. } \\
\text { Van de } \\
\text { Vyver, } \\
\text { T. L. } \\
\text { Vandoo } \\
\text { rn, B. } \\
\text { Meersm } \\
\text { an, J. } \\
\text { Desmet, } \\
\text { and L. } \\
\text { Vandev } \\
\text { elde }\end{array}$ & 2017 & {$[35]$} & $\begin{array}{l}\text { In this research, } \\
\text { charging } \\
\text { schedule for EV } \\
\text { participation to } \\
\text { improve system } \\
\text { security and } \\
\text { reduction in } \\
\text { emission cost is } \\
\text { presented. A } \\
\text { heuristics-based } \\
\text { optimization } \\
\text { methodology for } \\
\text { a day-ahead unit } \\
\text { commitment } \\
\text { (UC) model in } \\
\text { microgrids is } \\
\text { proposed. The } \\
\text { model aims to } \\
\text { schedule the } \\
\text { power among } \\
\text { the different } \\
\text { microgrid units } \\
\text { while } \\
\text { minimizing the } \\
\text { operating costs } \\
\text { together with the } \\
\text { CO2 emissions } \\
\text { produced }\end{array}$ & $\begin{array}{l}\text { Load } \\
\text { balance } \\
\text { constraint, } \\
\text { Emission } \\
\text { cost } \\
\text { constraint. }\end{array}$ \\
\hline M. Ban, & 2017 & [36] & In this research, & power \\
\hline
\end{tabular}




\begin{tabular}{|c|c|c|c|c|c|c|c|c|c|}
\hline \multirow[b]{2}{*}{$\begin{array}{l}\text { J. Yu, } \\
\text { M. } \\
\text { Shahide } \\
\text { hpour, } \\
\text { and Y. } \\
\text { Yao }\end{array}$} & & & \multirow[b]{2}{*}{$\begin{array}{l}\text { a solution for } \\
\text { imbalance } \\
\text { between supply } \\
\text { and demand is } \\
\text { provided by } \\
\text { using benders } \\
\text { decomposition } \\
\text { method. The } \\
\text { power } \\
\text { imbalance } \\
\text { between utility } \\
\text { and demand is } \\
\text { absolutely } \\
\text { handled by P2H } \\
\text { technology } \\
\text { which converts } \\
\text { excess wind } \\
\text { power by } \\
\text { electrolysis } \\
\text { process. The } \\
\text { ultimate result } \\
\text { of this } \\
\text { combination } \\
\text { with G2P } \\
\text { facilitate } \\
\text { accumulation of } \\
\text { excess wind } \\
\text { power. }\end{array}$} & \multirow[b]{2}{*}{$\begin{array}{l}\text { balance } \\
\text { constraints } \\
\text { ramping } \\
\text { up/down } \\
\text { limits, unit } \\
\text { generation } \\
\text { limits, } \\
\text { spinning } \\
\text { reserve }\end{array}$} & & \multirow[b]{2}{*}{2017} & \multirow[b]{2}{*}{ [39] } & \multirow[b]{2}{*}{$\begin{array}{l}\text { solved. } \\
\text { This paper } \\
\text { presents a new } \\
\text { algorithm called } \\
\text { as regrouping } \\
\text { swarm } \\
\text { optimization to } \\
\text { improve } \\
\text { performance by } \\
\text { simulating the } \\
\text { system over a } \\
\text { day and results } \\
\text { are recorded } \\
\text { with and without } \\
\text { wind penetration } \\
\text {.The system can } \\
\text { effectively use } \\
\text { for } \\
\text { commercializati } \\
\text { on of power. } \\
\text { Performance } \\
\text { objective } \\
\text { includes } \\
\text { minimization of } \\
\text { fuel cost, } \\
\text { operation and } \\
\text { maintenance } \\
\text { cost. }\end{array}$} & \multirow[b]{2}{*}{$\begin{array}{l}\text { Grid power } \\
\text { exchange } \\
\text { limits, } \\
\text { Demand- } \\
\text { supply } \\
\text { balance, ESS } \\
\text { units } \\
\text { charging/dis } \\
\text { charging } \\
\text { power limits, } \\
\text { ESS units } \\
\text { dynamic } \\
\text { operation } \\
\text { performance }\end{array}$} \\
\hline & & & & & $\begin{array}{l}\mathrm{H} . \mathrm{Li}, \\
\text { A. T. } \\
\text { Eseye, } \\
\text { J. } \\
\text { Zhang, } \\
\text { and D. } \\
\text { Zheng }\end{array}$ & & & & \\
\hline $\begin{array}{l}\text { S. } \\
\text { Chandra } \\
\text { shekar, } \\
\text { Y. Liu, } \\
\text { and R. } \\
\text { Sioshan } \\
\text { si }\end{array}$ & 2017 & [37] & $\begin{array}{l}\text { In this research, } \\
\text { benefits of } \\
\text { flexible } \\
\text { charging hours } \\
\text { on the wind } \\
\text { uncertainties } \\
\text { and variability is } \\
\text { given to reduce } \\
\text { the cost while } \\
\text { charging all } \\
\text { times. In this } \\
\text { article various } \\
\text { span of V2G } \\
\text { and G2G is } \\
\text { presented which } \\
\text { is to be } \\
\text { effectively } \\
\text { utilized so that } \\
\text { consumer can } \\
\text { earn more profit } \\
\text { from power sell. }\end{array}$ & $\begin{array}{l}\text { Ramp-up } \\
\text { constraints, } \\
\text { charger } \\
\text { capacity, } \\
\text { state- } \\
\text { transition } \\
\text { logic }\end{array}$ & $\begin{array}{l}\text { J. Meus, } \\
\text { K. } \\
\text { Poncele } \\
\text { t, and E. } \\
\text { Delarue }\end{array}$ & 2018 & {$[40]$} & $\begin{array}{l}\text { In this research, } \\
\text { fast } \\
\text { approximation } \\
\text { solution to UC } \\
\text { problem is } \\
\text { provided by } \\
\text { grouping similar } \\
\text { plants into } \\
\text { clusters such } \\
\text { that the binary } \\
\text { commitment } \\
\text { variables are } \\
\text { replaced by a } \\
\text { single integer } \\
\text { variable. This } \\
\text { grouping helps } \\
\text { to convert } \\
\text { binary } \\
\text { commitment } \\
\text { variables into a } \\
\text { single integer }\end{array}$ & $\begin{array}{l}\text { System } \\
\text { Constraints, } \\
\text { Technologic } \\
\text { al } \\
\text { constraints }\end{array}$ \\
\hline \multirow[t]{2}{*}{$\begin{array}{l}\text { A. } \\
\text { Babin, } \\
\text { N. } \\
\text { Rizoug, } \\
\text { T. } \\
\text { Mesbah } \\
\text { i, D. } \\
\text { Boscher } \\
\text {, Z. } \\
\text { Hamdo } \\
\text { un, and } \\
\text { C. } \\
\text { Larouci }\end{array}$} & \multirow[t]{2}{*}{2017} & \multirow[t]{2}{*}{ [38] } & \multirow{2}{*}{$\begin{array}{l}\text { In this research, } \\
\text { solution to } \\
\text { battery charging } \\
\text { for reducing the } \\
\text { cost has been } \\
\text { implemented in } \\
\text { a software tool. } \\
\text { Problems related } \\
\text { to aging, battery } \\
\text { sizing in } \\
\text { commercial } \\
\text { electric vehicle } \\
\text { (EV) using } \\
\text { intelligent } \\
\text { charging so as to } \\
\text { increase total } \\
\text { cost of } \\
\text { consumer are } \\
\text { effectively }\end{array}$} & \multirow[t]{2}{*}{$\begin{array}{l}\text { Charging } \\
\text { and } \\
\text { Discharging } \\
\text { Constraints }\end{array}$} & & & & $\begin{array}{l}\text { variable. This } \\
\text { method } \\
\text { combines CUC } \\
\text { with } \\
\text { conventional } \\
\text { UC to reduce } \\
\text { errors } \\
\text { introduced due } \\
\text { to problem } \\
\text { formulation and } \\
\text { grouping of non- } \\
\text { identical units } \\
\text { and provide a } \\
\text { feasible and } \\
\text { optimal solution. }\end{array}$ & \\
\hline & & & & & $\begin{array}{l}\text { J. Liu, } \\
\text { C. D. } \\
\text { Laird, J. } \\
\text { K. }\end{array}$ & 2018 & [41] & $\begin{array}{l}\text { In this research, } \\
\text { convergence } \\
\text { rate and solution } \\
\text { quality is }\end{array}$ & $\begin{array}{l}\text { Minimum } \\
\text { uptime and } \\
\text { downtime } \\
\text { constraints }\end{array}$ \\
\hline
\end{tabular}




\begin{tabular}{|c|c|c|c|c|c|c|c|c|c|}
\hline $\begin{array}{l}\text { Scott, J. } \\
\text { P. } \\
\text { Watson, } \\
\text { and A. } \\
\text { Castillo }\end{array}$ & & & $\begin{array}{l}\text { improved by } \\
\text { building an } \\
\text { algorithm. An } \\
\text { attempt is made } \\
\text { to find an global } \\
\text { optimal solution } \\
\text { but lacks in } \\
\text { capturing } \\
\text { desired } \\
\text { computational } \\
\text { time. However, } \\
\text { except } \\
\text { computational } \\
\text { time, the } \\
\text { algorithm } \\
\text { efficiently } \\
\text { solves the UC- } \\
\text { AC problem. }\end{array}$ & $\begin{array}{l}\text { spinning } \\
\text { reserve } \\
\text { constraint, } \\
\text { startup/shutd } \\
\text { own costs }\end{array}$ & $\begin{array}{l}\mathrm{Li} \text {, and } \\
\text { B. M. } \\
\text { Hodge }\end{array}$ & & & $\begin{array}{l}\text { which shows } \\
\text { mapping of } \\
\text { individual unit } \\
\text { commitment } \\
\text { status with } \\
\text { CCGTs. The } \\
\text { hybrid model } \\
\text { consists of } \\
\text { combined-cycle } \\
\text { gas turbines } \\
\text { which provides } \\
\text { excellent } \\
\text { operational } \\
\text { flexibility, better } \\
\text { response to } \\
\text { sustainable } \\
\text { sources, ability } \\
\text { to generate more }\end{array}$ & $\begin{array}{l}\text { ramping } \\
\text { rates }\end{array}$ \\
\hline \multirow{2}{*}{$\begin{array}{l}\text { F. H. } \\
\text { Aghda } \\
\mathrm{m} \text { and } \\
\text { M. T. } \\
\text { Hagh }\end{array}$} & \multirow[t]{2}{*}{2018} & \multirow[t]{2}{*}[42]{} & \multirow[b]{2}{*}{$\begin{array}{l}\text { In this research, } \\
\text { problems of } \\
\text { transmission } \\
\text { lines and } \\
\text { operation } \\
\text { constraints } \\
\text { including } \\
\text { MUT/MDT and } \\
\text { emission limits } \\
\text { are analyzed } \\
\text { using MICA } \\
\text { algorithm. In } \\
\text { this method, } \\
\text { basic algorithm } \\
\text { uses priority list } \\
\text { (PL) method to } \\
\text { define initial } \\
\text { state. The } \\
\text { system is tested } \\
\text { on IEEE-30 bus } \\
\text { and } 118 \text {-bus } \\
\text { system }\end{array}$} & \multirow{2}{*}{$\begin{array}{l}\text { Minimum } \\
\text { uptime and } \\
\text { downtime } \\
\text { constraints } \\
\text { spinning } \\
\text { reserve } \\
\text { constraint, } \\
\text { startup/shutd } \\
\text { own costs }\end{array}$} & & & & $\begin{array}{l}\text { power compare } \\
\text { to conventional } \\
\text { method. }\end{array}$ & \\
\hline & & & & & $\begin{array}{l}\text { C. Zhu, } \\
\text { F. Lu, } \\
\text { H. } \\
\text { Zhang, } \\
\text { and C. } \\
\text { C. Mi }\end{array}$ & 2018 & {$[45]$} & $\begin{array}{l}\text { In this research, } \\
\text { temperature } \\
\text { sensitive nature } \\
\text { of lithium-ion } \\
\text { batteries, finite } \\
\text { robust predictive } \\
\text { strategy is } \\
\text { presented for } \\
\text { reduce heat } \\
\text { effect to damage } \\
\text { system. Further } \\
\text { proposed } \\
\text { method facilitate } \\
\text { to predict future } \\
\text { temperature } \\
\text { forecast and } \\
\text { enables to } \\
\text { provide } \\
\text { flexibility to }\end{array}$ & $\begin{array}{l}\text { Heating and } \\
\text { cooling } \\
\text { constraints, } \\
\text { Unit } \\
\text { generation } \\
\text { limits }\end{array}$ \\
\hline \multirow{3}{*}{$\begin{array}{l}\text { A. } \\
\text { Yazdan } \\
\text { doost, } \\
\text { P. } \\
\text { Khazaei } \\
\text {, R. } \\
\text { Kamali, } \\
\text { and S. } \\
\text { Saadatia } \\
\text { n }\end{array}$} & \multirow[t]{3}{*}{2018} & \multirow[t]{3}{*}[43]{} & \multirow{3}{*}{$\begin{array}{l}\text { In this research, } \\
\text { generating } \\
\text { schedule is } \\
\text { controlled to } \\
\text { minimize the } \\
\text { total operating } \\
\text { cost by } \\
\text { introducing } \\
\text { modified genetic } \\
\text { algorithm based } \\
\text { on multicellular } \\
\text { organism's } \\
\text { mechanisms. } \\
\text { This algorithm } \\
\text { combines the } \\
\text { features of GA } \\
\text { and modified } \\
\text { GAMOM. The } \\
\text { effectiveness of } \\
\text { the scheme is } \\
\text { validated } \\
\text { through the } \\
\text { nature of } \\
\text { convergence } \\
\text { curve. }\end{array}$} & \multirow{3}{*}{$\begin{array}{l}\text { Power } \\
\text { Balance, } \\
\text { Generating } \\
\text { Units } \\
\text { limitation, } \\
\text { Ramp up } \\
\text { and down } \\
\text { limitation, } \\
\text { Startup and } \\
\text { shutdown } \\
\text { Constraints, } \\
\text { Voltage and } \\
\text { Angle } \\
\text { constraints. }\end{array}$} & & & & driving profiles. & \\
\hline & & & & & $\begin{array}{l}\text { S. } \\
\text { Maghsu } \\
\text { dlu and } \\
\text { S. } \\
\text { Moham } \\
\text { madi }\end{array}$ & 2018 & [46] & $\begin{array}{l}\text { This research } \\
\text { presents a novel } \\
\text { stochastic } \\
\text { Monte Carlo } \\
\text { optimization } \\
\text { algorithm to } \\
\text { handle } \\
\text { uncertainties of } \\
\text { solar power. } \\
\text { This study } \\
\text { includes the } \\
\text { investigations in } \\
\text { presence of EV } \\
\text { and PV. Also, } \\
\text { an efficient } \\
\text { balance is seen } \\
\text { between } \\
\text { uncertain energy } \\
\text { source and EV } \\
\text { system. }\end{array}$ & $\begin{array}{l}\text { Generation } \\
\text { limits, } \\
\text { Vehicle } \\
\text { balance }\end{array}$ \\
\hline & & & & & $\begin{array}{l}\text { X. } \\
\text { Chen, } \\
\text { M. B. } \\
\text { Mcelroy }\end{array}$ & 2018 & [47] & $\begin{array}{l}\text { This paper } \\
\text { presents } \\
\text { practical barriers } \\
\text { while dealing }\end{array}$ & $\begin{array}{l}\text { Penetration } \\
\text { level of } \\
\text { renewables, } \\
\text { Emission }\end{array}$ \\
\hline $\begin{array}{l}\text { X. } \\
\text { Fang, L. } \\
\text { Bai, F. }\end{array}$ & 2018 & {$[44]$} & $\begin{array}{l}\text { In this paper, a } \\
\text { hybrid model is } \\
\text { developed }\end{array}$ & $\begin{array}{l}\text { Minimum } \\
\text { online/offlin } \\
\text { e time and }\end{array}$ & $\begin{array}{l}\text {, Q. } \\
\text { Wu, Y. } \\
\text { Shu, }\end{array}$ & & & $\begin{array}{l}\text { with operation } \\
\text { of collaborative } \\
\text { functioning of }\end{array}$ & Constraints \\
\hline
\end{tabular}




\begin{tabular}{|c|c|c|c|c|}
\hline $\begin{array}{l}\text { and Y. } \\
\text { Xue }\end{array}$ & & & $\begin{array}{l}\text { uncertain } \\
\text { distributed } \\
\text { generation and } \\
\text { simultaneously } \\
\text { maintaining } \\
\text { emission and } \\
\text { environmental } \\
\text { constraints } \\
\text { within limit. }\end{array}$ & \\
\hline $\begin{array}{l}\text { T. K. } \\
\text { Renuka, } \\
\text { P. Reji, } \\
\text { and S. } \\
\text { Sreedha } \\
\text { ran, }\end{array}$ & 2018 & [48] & $\begin{array}{l}\text { This paper } \\
\text { presents a } \\
\text { multistage PSO } \\
\text { method for } \\
\text { improving both } \\
\text { the energy } \\
\text { penetration and } \\
\text { small signal } \\
\text { stability. This } \\
\text { method is tested } \\
\text { on IEEE 14-bus } \\
\text { at } 220 \text { KV } \\
\text { practical system } \\
\text { with the solar } \\
\text { and wind power. } \\
\text { The program is } \\
\text { carried out in } \\
\text { two stages. In } \\
\text { first stage } \\
\text { renewable } \\
\text { penetration is } \\
\text { addressed while } \\
\text { in second stage } \\
\text { stability issue is } \\
\text { handled. }\end{array}$ & $\begin{array}{l}\text { Equality } \\
\text { constraints, } \\
\text { Inequality } \\
\text { constraints, } \\
\text { Fast voltage } \\
\text { stability } \\
\text { indices, Line } \\
\text { stability } \\
\text { index }\end{array}$ \\
\hline $\begin{array}{l}\text { T. } \\
\text { Ghose, } \\
\text { H. } \\
\text { W.Pand } \\
\text { ey, K. } \\
\text { R. } \\
\text { Gadham }\end{array}$ & 2019 & $\begin{array}{l}49] \\
\end{array}$ & $\begin{array}{l}\text { This paper } \\
\text { describes about } \\
\text { two risk } \\
\text { assessment } \\
\text { techniques } \\
\text { which predicts } \\
\text { the option of } \\
\text { providing power } \\
\text { either according } \\
\text { to demand } \\
\text { response or } \\
\text { renewable } \\
\text { energy source. } \\
\text { The main } \\
\text { objective of this } \\
\text { research is to } \\
\text { focus mainly on } \\
\text { financial risk of } \\
\text { aggregators } \\
\text { while } \\
\text { maintaining the } \\
\text { conditions of } \\
\text { technical aspects } \\
\text { of microgrids. }\end{array}$ & $\begin{array}{l}\text { Minimum } \\
\text { and } \\
\text { maximum } \\
\text { capacity of } \\
\text { the DR, } \\
\text { minimum } \\
\text { and } \\
\text { maximum } \\
\text { durations of } \\
\text { load } \\
\text { recovery. }\end{array}$ \\
\hline $\begin{array}{l}\text { M. } \\
\text { Bayati, } \\
\text { M. } \\
\text { Abedi, } \\
\text { G. B. } \\
\text { Ghareh } \\
\text { petian, } \\
\text { and M. } \\
\text { Farahm } \\
\text { andrad } \\
\end{array}$ & 2019 & {$[50]$} & $\begin{array}{l}\text { In this research, } \\
\text { EV power } \\
\text { output effects on } \\
\text { the conventional } \\
\text { fuel cost is } \\
\text { presented in } \\
\text { very lucid } \\
\text { manner. Also, } \\
\text { benefits of EV } \\
\text { technologies are }\end{array}$ & $\begin{array}{l}\text { Power } \\
\text { balance, } \\
\text { Charging } \\
\text { /Discharging } \\
\text { constraints }\end{array}$ \\
\hline
\end{tabular}

\begin{tabular}{|c|c|c|c|c|}
\hline & & & $\begin{array}{l}\text { properly utilized } \\
\text { to tackle } \\
\text { problems related } \\
\text { to } \\
\text { environmental } \\
\text { pollution. } \\
\text { Frequency } \\
\text { deviation due to } \\
\text { EV power } \\
\text { output is } \\
\text { handled by } \mathrm{V} 2 \mathrm{G} \\
\text { technology. }\end{array}$ & \\
\hline $\begin{array}{l}\text { J. B. } \\
\text { Mogo } \\
\text { and I. } \\
\text { Kamwa }\end{array}$ & 2019 & {$[51]$} & $\begin{array}{l}\text { This paper } \\
\text { presents a } \\
\text { security } \\
\text { constraint unit } \\
\text { commitment is } \\
\text { implemented } \\
\text { and effectively } \\
\text { reduces burden } \\
\text { of spinning } \\
\text { reserve on } \\
\text { spinning reserve } \\
\text { requirement on } \\
\text { conventional } \\
\text { sources by } \\
\text { utilizing wind } \\
\text { power as a } \\
\text { reserve using } \\
\text { mixed integer } \\
\text { programming } \\
\text { thereby reducing } \\
\text { the overall } \\
\text { operating cost. }\end{array}$ & $\begin{array}{l}\text { Ramping } \\
\text { reserve } \\
\text { limits, } \\
\text { spinning } \\
\text { reserve } \\
\text { capacity } \\
\text { limit. }\end{array}$ \\
\hline $\begin{array}{l}\text { Z. } \\
\text { Yang, } \\
\text { K. Li, } \\
\text { Y. Guo } \\
\text {, S. } \\
\text { Feng, } \\
\text { Q. Niu, } \\
\text { Y. Xue, } \\
\text { A.Foley }\end{array}$ & 2019 & [6] & $\begin{array}{l}\text { The paper } \\
\text { presents a binary } \\
\text { symmetric } \\
\text { method which } \\
\text { solves the } \\
\text { complexity of } \\
\text { hybrid system in } \\
\text { presence of } \\
\text { uncertain } \\
\text { sources and } \\
\text { electric vehicles. } \\
\text { Particle swarm } \\
\text { optimization } \\
\text { algorithm is } \\
\text { utilized to } \\
\text { provide flexible } \\
\text { charging/dischar } \\
\text { ging patterns } \\
\text { thereby reducing } \\
\text { the cost of } \\
\text { generation and } \\
\text { emission. }\end{array}$ & $\begin{array}{l}\text { Generation } \\
\text { limit, Power } \\
\text { demand } \\
\text { limit, Power } \\
\text { reserve limit, } \\
\text { Minimum } \\
\text { up/down } \\
\text { time limit }\end{array}$ \\
\hline
\end{tabular}

\section{MATHEMATICAL MODELING}

The objective of unit commitment is to plan the optimal schedule of available generating units to minimize the total operational and generation cost. Total cost of power generation includes fuel cost, shut down and start-up costs within constraints limit.

The various constriants are as follows: 


\subsection{Operating Cost}

The operating cost of $i^{\text {th }}$ at $h^{\text {th }}$ hour and can be represented as below:

$$
F_{h i}=\left(a_{i} P_{h i}^{2}+b_{i} P_{h i}+c_{i}\right) U_{h i}+S U C_{h i}\left(1-U_{(h-1) i}\right) U_{h i}
$$

$$
(i=1,2, \ldots, N G ; h r=1,2, \ldots, H)
$$

Where, Fhi is the cost associated with the $i^{\text {th }}$ generating unit at $\mathrm{h}^{\text {th }}$ hour and $a_{i}, b_{i}$ and $c_{i}$ are its fuel and operational cost coefficients, $U_{h i}$ and $U_{(h-1) i}$ is the committed status of the ith unit at $\mathrm{h}^{\text {th }}$ hour and (h-1)-th hour respectively, $\mathrm{SUC}_{\mathrm{hi}}$ is the start-up cost of $i^{\text {th }}$ unit at $\mathrm{h}^{\text {th }}$ hour

Combined cost $\left(\mathrm{F}_{\mathrm{h}}\right)$, for all the generating units $(\mathrm{NG})$ at a particular hour ' $h$ ' can be obtained as the sum total of all the individual units 'costs:

$$
F_{h r}=\sum_{i=1}^{N G}\left[\left(a_{i} P_{h i}^{2}+b_{i} P_{h i}+c_{i}\right) U_{h i}+S U C_{h i}\left(1-U_{(h-1) i}\right) U_{h i}\right] \sum_{i=1}^{N G} P_{h r i} U_{h r i}+P_{h r}^{\text {Sustainable }=} D_{h r}+D_{h r}^{\text {Vehicle }} \quad(h r=1,2, \ldots, H)
$$

\section{Load Balance Constraints}

The load balance or system power balance constraint requires that the sum of generation of all the committed units at hth hour must be greater than or equal to the demand $\mathrm{Dh}$ at a particular hour ' $h$ '.

$$
\sum_{i=1}^{N G} P_{h r i} U_{h r i}+P_{h r}^{\text {Sustainable }}=D_{h r} \quad(h r=1,2, \ldots, H)
$$

\section{Case-1: During Charging of Vehicle}

\section{Case-2: During Discharging}

$\sum_{i=1}^{N G} P_{h r i} U_{h r i}+P_{h r}^{\text {Sustainable }}+D_{h r}^{\text {Vehicle }}=D_{h r} \quad(h r=1,2, \ldots, H)$

Now, the total fuel cost $\mathrm{F}$ is the double summation of the costs incurred for all the generators for all the time periods considered. It can be mathematically represented as:

$$
F=\sum_{h=1}^{H}\left(\sum_{i=1}^{N G}\left[\left(a_{i} P_{h i}^{2}+b_{i} P_{h i}+c_{i}\right) U_{h i}+S U C_{h i}\left(1-U_{(h-1) i}\right) U_{h i}\right]\right)
$$$$
(\mathbf{z} / \mathbf{h})(\mathrm{hr}=1,2 \ldots \mathrm{H})
$$

Above eqn.(4) does not contain power loss in the system. If hourly power loss $\mathrm{Ph}_{\mathrm{L}}$ is considered, then eqn.(4) can be modified as:

$$
\sum_{i=1}^{N G} P_{h r i} U_{h r i}+P_{h r}^{\text {Sustainable }}=D_{h} r+P_{h r L} \quad(h r=1,2, \ldots, H)(5 \mathrm{a})
$$

Mathematically, startup cost $\left(S U C_{h i}\right)$ can be expressed as:

$$
\begin{aligned}
& \operatorname{Sup}_{h i}= \begin{cases}H S C_{i} ; & M D t_{i} \leq T_{h i}^{O F F} \leq\left(M D t_{i}+C s H_{i}\right) \\
C S c_{i} ; & T_{h i}^{O F F}>\left(M D T_{i}+C s H_{i}\right)\end{cases} \\
& (i=1,2, \ldots, N G ; h r=1,2, \ldots, H)
\end{aligned}
$$

where, $C S c_{i}$ and $H S C_{i}$ are cold startup and hot start-up cost of i-th unit respectively and $M D t_{i}$ is the minimum down time of i-th unit, $T_{h i}^{O F F}$ is duration for which the i-th thermal unit has been continuously off until hour $h$. $\mathrm{CSH}_{i}$ is the cold start hour of $\mathrm{i}$-th unit. The start-up cost for a unit depends on its downtime. If it is longer than the related MDt plus its predefined Cold-Start Hours $(\mathrm{CsH})$, Cold-Start Cost (CSc) is needed to operate it. Else if the ith unit down time is shorter than the mentioned duration, Hot-Start cost (HSC) is needed to operate it. The Various Constraints linked with unit commitment problem are explained below.

\section{Case-1: During Charging of Vehicle}

$\sum_{i=1}^{N G} P_{h r i} U_{h r i}+P_{h r}^{\text {Sustainable }}=D_{h r}+P_{h r L}+D_{h}^{\text {Vehicle }} \quad(h r=1,2, \ldots, H)$

\section{Case-2: During Discharging}

$\sum_{i=1}^{N G} P_{h r i} U_{h r i}+P_{h r}^{\text {Sustainable }}+D_{h r}^{\text {Vehicle }}=D_{h r}+P_{h r L} \quad(h r=1,2, \ldots, H)$ (5c)

The power generation of the NG generating units at a particular time horizon handles theload balance constraint and other operating limit constraints. To satisfy the equality constraints, one unit is designated as reference unit and its power generation is decided as follows: For any arbitrarily available unit output power generation( Phi), $P_{i(\min )} \leq P_{h r i} \leq P_{i(\max )},(\mathrm{i}=1,2, \ldots, \mathrm{NG})$, it is assumed that power output available at the Rth unit $t$ is constrained by the load(power) balance equation as: 
$P_{h r R}=D_{h r}+P_{h r L}-\sum_{\substack{i=1 \\ i \neq R}}^{N G}\left(P_{h r i}+P_{h r}^{\text {Sustainable }}\right) \quad(h r=1,2, \ldots, H)$

\section{Case-1: During Charging of Vehicle}

$P_{h r R}=D_{h r}+P_{h r L}-\sum_{\substack{i=1 \\ i \neq R}}^{N G}\left(P_{h r i}+P_{h r}^{\text {Sustainable }}-D_{h r}^{\text {Vehicle }}\right) \quad(h r=1,2, \ldots, H)$

(6b) Case-2: During Discharging

$P_{h r R}=D_{h r}+P_{h r L}-\sum_{\substack{i=1 \\ i \neq R}}^{N G}\left(P_{h r i}+P_{h r}^{\text {Sustainable }}+D_{h r}^{\text {Veh }}\right) \quad(h r=1,2, \ldots, H)$

(6c)

\section{Reserve Power (Spinning) Constraints}

To deal with unpredictable disturbances (interruption of generation and transmission lines or unexpected increase in demand) certain amount of reserve capacity must be always available. This excess capacity of generation is known as Spinning Reserve $(R s)$ and mathematically given as:

$\sum_{i=1}^{N G} P_{i(\max )} U_{h i}+P_{h r}^{\text {Sustainable }} \geq D_{h r}+R_{s h r} \quad(h r=1,2, \ldots, H)$

\section{Case-1: During Charging of Vehicle}

$$
\sum_{i=1}^{N G} P_{i(\max )} U_{h r i}+P_{h r}^{\text {Sustanabble }} \geq D_{h r r}+R_{s l r}+D_{h r}^{\text {vehicle }} \quad(h r=1,2, \ldots, H)
$$

\section{Case-2: During Discharging}

$$
\sum_{i=1}^{N G} P_{i(\max )} U_{h i}+P_{h r}^{\text {Sustainable }} \geq D_{h r}+R_{s h l}-D_{h r}^{\text {Velicle }} \quad(h r=1,2, \ldots, H)(7 \mathrm{c})
$$

\section{Thermal Constraints}

A thermal constraint includes (a) minimum up time i.e. time required to turn-on a unit from shunt down condition (b) Minimum down time i.e. time required to turn -off already running units and (c) crew constraints i.e. limitations of crew members to attend more than one unit at the same time. These constraints may result in many hurdles in the operation of thermal units.

\section{Minimum-up Time}

Mathematically expressed as:

$$
T_{h i}^{O N} \geq M_{U} t_{i} \quad(i=1,2, \ldots, N G ; h r=1,2, \ldots, H) \cdot
$$

Where, $T_{h i}^{O N}$ is time interval for which ith unit is continuously $\mathrm{ON}$ (in hrs) and $M U t_{i}$ is its minimum up time (in hrs).

\section{Minimum-down Time}

Mathematically expressed as:

$$
T_{h r i}^{\text {OFF }} \geq M D t_{i} \quad(i=1,2, \ldots, N G ; h r=1,2, \ldots, H)(8 \mathrm{~b})
$$

where, $T_{h r i}^{\text {OFF }}$ is time interval for which ith unit is continuously OFF (in hrs) and $M D t_{i}$ is it's minimum down time (in hrs).

\section{Crew Constraints}

If a plant consists of two or more units, there may not be enough crewmembers to attend all the units simultaneously while starting up.

\section{Initial Operating Status of Generating Units}

Its decides minimum up /down time satisfaction of every unit depending upon data of last day's previous schedule

\section{Constraints Repairing Mechanism}

The flowchart for constraint handling mechanism during charging and discharging phase has been depicted below. The Fig.1 shows the constraint handling mechanism for UCP minimum up and down time. And Fig. 2 represents the constraints handling mechanism for spinning reserve requirements.

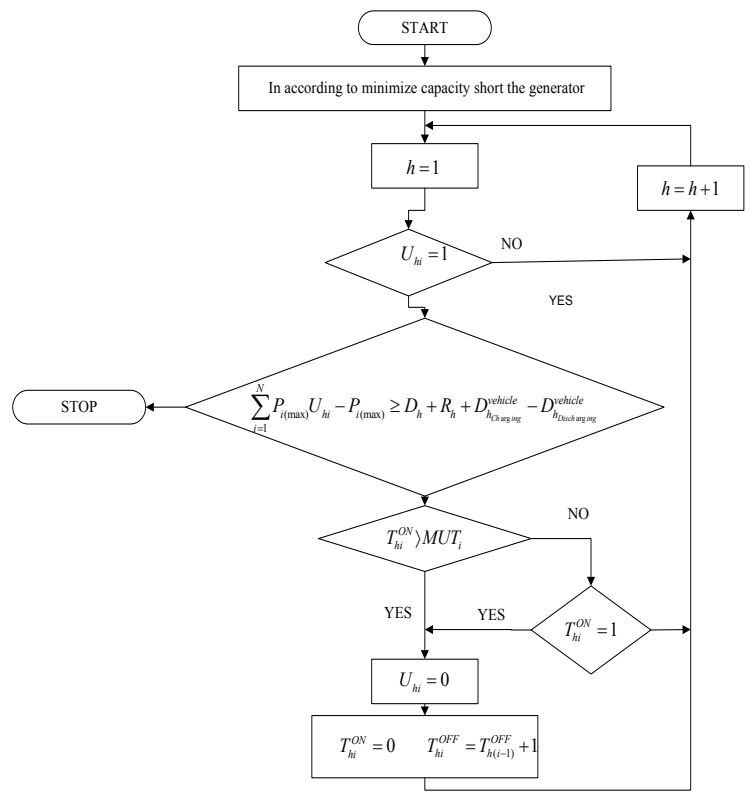

Fig.1: Handling of MDT and MUT during charging and 




Fig.2: Fulfillment of spinning reserve requirements during charging and Discharging Phase

\section{SCOPE OF RESEARCH}

The analysis of present review suggest that UC problem is aimed at determining the turn-On and turn -off schedules of thermal units to meet forecasted demand for a certain time interval and belongs to a combinatorial optimization problem. Further, it is clear that optimization falls roughly into three categories: heuristic search, mathematical programing, and hybrid methods. There are several optimization strategies employed to solve the complexity of generation scheduling and dispatch problem. Some of these methods are the, Bat Algorithm, Binary Gravitational Search Algorithm, Backtracking Search Optimization, Colliding Bodies Optimization, , Chaotic Krill Herd Algorithm, Dynamic Programming, Dragonfly Algorithm, Fireworks Algorithm, Flower Pollination Algorithm, Grey Wolf Optimizer, Imperialist Competitive Algorithm, Mine Blast Algorithm, Optics Inspired Optimization, Random Walk Grey Wolf Optimizer, Simulated Annealing, Sine Cosine Algorithm, Search Group Algorithm, Symbiotic Organisms Search, Salp Swarm Algorithm, Virus Colony Search, Water Cycle Algorithm, Water Wave Optimization, etc.

From the research paper listed above in Table-1 and research problem, it have been seen that great efforts are taken for achieving economic load dispatch and unit commitment problem using different methodologies, but there needs some more considerable efforts are made to find solution for global optimized solution (within local and global search space) for cost effective solution for unit commitment problem for vehicle to grid operation in sustainable energy environment, which seriously affects the optimality of the results. Also, it is clear from the literatures, appreciable efforts are made to solve the unit commitment problem using various meta-heuristics optimization methodologies, but no significant efforts are made to find out the global optimization search algorithm by combining local and global search capability of algorithm to get more improved results. Further, research theorem has logically suggested that none of the optimization algorithm is able to provide exact solution to all types of optimization problems efficiently. In other words, there is always scope of improvements to upgrade current techniques to better solve maximum optimization problems efficiently.

In recent research studies pertaining to optimization algorithm, it has been reported that swarm intelligence optimization have some drawbacks and need advanced solutions. Another important concern in swarm intelligent algorithm is regarding exploration, exploitation and convergence. In practical application, it creates serious problem. This motivated our attempts to propose yet another memetic solution of with due consideration of sustainable energy sources along with vehicle to grid concept.

\section{Conclusion And Future Scope}

In the proposed research, the authors has successfully presented the scope of solution intelligence solution strategies for effective vehicle to grid operation for unit commitment problem with due consideration of sustainable energy sources. Further, Electric vehicles are getting popularity due to eco-friendly nature and could be a part of power sector in the future. Thus, proposed research explores the concept of modern smart grid system along with effective solution strategies. It is recommended that, in order to explore the scope of intelligence approaches in future learning, the following future work may be taken into consideration:

- Consideration of memetic intelligence approaches for cost effective solution of unit-commitment due consideration of uncertainty of sustainable power and vehicle to grid operations.

- Development of hybrid optimization algorithm by combing local search algorithm with modern global search algorithm for constrained optimization problem can be explored and its performance testing can be made on various standard unimodal, multi model, fixed dimension and engineering benchmark problems.

- Simulation environment can be created for implementation of unit commitment problem of electric power system with due consideration of Vehicle to Grid operation in stochastic sustainable environment energy environment 
- Testing and validation of proposed memetic intelligence algorithm can be explored for various IEEE benchmarks problems with incorporation of V2G operations in stochastic sustainable environment.

\section{References}

1. A. Bhadoria, V. K. Kamboj, M. Sharma, and S. K. Bath, "A Solution to Non-convex/Convex and Dynamic Economic Load Dispatch Problem Using Moth Flame Optimizer," Ina. Lett., vol. 3, no. 2, pp. 65-86, (2018).

2. M. Esmaeeli, S. Golshannavaz, and P. Siano, "Determination of optimal reserve contribution of thermal units to afford the wind power uncertainty," J. Ambient Intell. Humaniz. Comput., vol. 11, no. 4, pp. 1565-1576, (2020).

3. C. De Jonghe, E. Delarue, R. Belmans, and W. D'haeseleer, "Determining optimal electricity technology mix with high level of wind power penetration," Appl. Energy, vol. 88, no. 6, pp. 2231-2238, (2011).

4. M. S. Shahriar, M. J. Rana, M. A. Asif, M. M. Hasan, and M. M. Hawlader, "Optimization of Unit Commitment Problem for wind-thermal generation using Fuzzy optimization technique," in Proceedings of 2015 3rd International Conference on Advances in Electrical Engineering, ICAEE 2015, pp. 88-92(2015).

5. M. Wang et al., "A preventive control strategy for static voltage stability based 92(2016on an efficient power plant model of electric vehicles," J. Mod. Power Syst. Clean Energy, vol. 3, no. 1, pp. 103-113, (2015).

6. Z. Yang et al., "A binary symmetric based hybrid meta-heuristic method for solving mixed integer unit commitment problem integrating with significant plug-in electric vehicles," Energy, vol. 170, pp. 889-905,(2019).

7. W. L. Snyder, H. D. Powell, and J. C. Rayburn, "Dynamic Programming Approach to Unit Commitment," IEEE Power Eng. Rev., vol. PER7, no. 5, pp. 41-42, (1987).

8. S. Virmani, E. Adrian, ... K. I.-I. T. on, and undefined 1989, "Implementation of a Lagrangian relaxation based unit commitment problem," ieeexplore.ieee.org (1989)

9. V. K. Kamboj, S. K. Bath, and J. S. Dhillon, "Implementation of hybrid harmony/random search algorithm considering ensemble and pitch violation for unit commitment problem," Int. J. Electr. Power Energy Syst., vol. 77, pp. 228-249, (2016).

10. A. Y. Saber and G. K. Venayagamoorthy, "Unit commitment with vehicle-to-grid using particle swarm optimization," 2009 IEEE Bucharest PowerTech Innov. Ideas Towar. Electr. Grid Futur., pp. 1-8, (2009).

11. S. A. Kazarlis, "A genetic algorithm solution to the unit commitment problem", IEEE Transactions on
Power Systems, pp. 83 -92, (1996).

12. V. N. Dieu and W. Ongsakul, "Ramp rate constrained unit commitment by improved priority list and augmented Lagrange Hopfield network," Electr. Power Syst. Res., vol. 78, no. 3, pp. 291301, (2008).

13. M. Singh, I. Kar, and P. Kumar, "Influence of EV on grid power quality and optimizing the charging schedule to mitigate voltage imbalance and reduce power loss," Proc. EPE-PEMC 2010 - 14th Int. Power Electron. Motion Control Conf., pp. 196203, (2010).

14. B. Palmintier and M. Webster, "Impact of unit commitment constraints on generation expansion planning with renewables," IEEE Power Energy Soc. Gen. Meet., pp. 1-7,(2011).

15. S. Kamboj, W. Kempton, and K. S. Decker, "Deploying Power Grid-Integrated Electric Vehicles as a Multi-Agent System," Proc. 10th Int. Conf. Auton. Agents Multiagent Syst. - Innov. Appl. Track (AAMAS 2011), no. Aamas, pp. 1320, (2011).

16. G. O. Suvire, M. G. Molina, and P. E. Mercado, "Improving the integration of wind power generation into AC microgrids using flywheel energy storage," IEEE Trans. Smart Grid, vol. 3, no. 4, pp. 1945-1954, (2012).

17. A. Foley, B. Tyther, P. Calnan, and B. Ó Gallachóir, "Impacts of Electric Vehicle charging under electricity market operations," Appl. Energy, vol. 101, no. 2013, pp. 93-102, (2013).

18. P. K. Roy, "Solution of unit commitment problem using gravitational search algorithm," Int. J. Electr. Power Energy Syst., vol. 53, no. 1, pp. 85-94, (2013).

19. L. Liu, H. Li, Y. Xue, and W. Liu, "Reactive power compensation and optimization strategy for grid-interactive cascaded photovoltaic systems," IEEE Trans. Power Electron., vol. 30, no. 1, pp. 188-202, (2015).

20. M. E. El-Hawary, "The smart grid - State-of-theart and future trends," Electr. Power Components Syst., vol. 42, no. 3-4, pp. 239-250, (2014).

21. P. B. Luh et al., "Grid Integration of Distributed Wind Generation: Hybrid Markovian and Interval Unit Commitment," IEEE Trans. Smart Grid, vol. 5, no. 2, pp. 732-741, (2014).

22. V. K. Kamboj, S. K. Bath, and J. S. Dhillon, "A novel hybrid DE-random search approach for unit commitment problem," Neural Comput. Appl., vol. 28, no. 7, pp. 1559-1581, (2017).

23. C. CHEN and S. DUAN, "Microgrid economic operation considering plug-in hybrid electric vehicles integration," J. Mod. Power Syst. Clean Energy, vol. 3, no. 2, pp. 221-231,( 2015).

24. H. LIU, P. ZENG, J. GUO, H. WU, and S. GE, "An optimization strategy of controlled electric vehicle charging considering demand side response and regional wind and photovoltaic," J. Mod. Power Syst. Clean Energy, vol. 3, no. 2, pp. 232-239, (2015).

25. S. Umamaheswaran and S. Rajiv, "Financing large 
scale wind and solar projects - A review of emerging experiences in the Indian context," Renew. Sustain. Energy Rev., vol. 48, pp. 166177, (2015).

26. N. Zhang, Z. Hu, X. Han, J. Zhang, and Y. Zhou, "A fuzzy chance-constrained program for unit commitment problem considering demand response, electric vehicle and wind power," Int. J. Electr. Power Energy Syst., vol. 65, pp. 201-209, (2015).

27. K. S. Reddy, L. K. Panwar, and R. Kumar, "Potential benefits of electric vehicle deployment as responsive reserve in unit commitment," 9th Int. Conf. Ind. Inf. Syst. ICIIS 2014, (2015).

28. L. Yang, J. Jian, Z. Dong, and C. Tang, "MultiCuts Outer Approximation Method for Unit Commitment," IEEE Trans. Power Syst., vol. 32, no. 2, pp. 1587-1588,(2017).

29. W. S. Tan and M. Shaaban, "A Hybrid Stochastic/Deterministic Unit Commitment Based on Projected Disjunctive MILP Reformulation," IEEE Trans. Power Syst., vol. 31, no. 6, pp. 52005201, 2016.

30. R. Á. Fernández, F. B. Cilleruelo, and I. V. Martínez, "A new approach to battery powered electric vehicles: A hydrogen fuel-cell-based range extender system," Int. J. Hydrogen Energy, vol. 41, no. 8, pp. 4808-4819, (2016).

31. K. S. Reddy, L. K. Panwar, R. Kumar, and B. K. Panigrahi, "Distributed resource scheduling in smart grid with electric vehicle deployment using fireworks algorithm," J. Mod. Power Syst. Clean Energy, vol. 4, no. 2, pp. 188-199, (2016).

32. V. Monteiro, J. G. Pinto, and J. L. Afonso, "Operation Modes for the Electric Vehicle in Smart Grids and Smart Homes: Present and Proposed Modes," IEEE Trans. Veh. Technol., vol. 65, no. 3, pp. 1007-1020, (2016).

33. Srinivas Rao J., Srinivasa Varma, P., Suresh Kumar. T, International Journal of Power Electronics and Drive Systems, vol.9, no.3, pp. 1202-1213, (2018).

34. E. S. Ali, S. M. Abd Elazim, and A. Y. Abdelaziz, "Ant Lion Optimization Algorithm for renewable Distributed Generations," Energy, vol. 116, pp. 445-458, (2016).

35. C. Deckmyn, J. Van de Vyver, T. L. Vandoorn, B. Meersman, J. Desmet, and L. Vandevelde, "Dayahead unit commitment model for microgrids," IET Gener. Transm. Distrib., vol. 11, no. 1, pp. 19, (2017).

36. M. Ban, J. Yu, M. Shahidehpour, and Y. Yao, "Integration of power-to-hydrogen in day-ahead security-constrained unit commitment with high wind penetration," J. Mod. Power Syst. Clean Energy, vol. 5, no. 3, pp. 337-349, (2017).

37. S. Chandrashekar, Y. Liu, and R. Sioshansi, "Wind-integration benefits of controlled plug-in electric vehicle charging," J. Mod. Power Syst. Clean Energy, vol. 5, no. 5, pp. 746-756, (2017).

38. A. Babin, N. Rizoug, T. Mesbahi, D. Boscher, Z. Hamdoun, and C. Larouci, "Total Cost of
Ownership Improvement of Commercial Electric Vehicles Using Battery Sizing and Intelligent Charge Method," IEEE Trans. Ind. Appl., vol. 54, no. 2, pp. 1691-1700, (2018).

39. H. Li, A. T. Eseye, J. Zhang, and D. Zheng, "Optimal energy management for industrial microgrids with high-penetration renewables," Prot. Control Mod. Power Syst., vol. 2, no. 1, pp. 1-14, (2017).

40. J. Meus, K. Poncelet, and E. Delarue, "Applicability of a Clustered Unit Commitment Model in Power System Modeling," IEEE Trans. Power Syst., vol. 33, no. 2, pp. 2195-2204, (2018).

41. J. Liu, C. D. Laird, J. K. Scott, J. P. Watson, and A. Castillo, "Global Solution Strategies for the Network-Constrained Unit Commitment Problem with AC Transmission Constraints," IEEE Trans. Power Syst., vol. 34, no. 2, pp. 1139-1150, (2019).

42. F. H. Aghdam and M. T. Hagh, "Security Constrained Unit Commitment (SCUC)formulation and its solving with Modified Imperialist Competitive Algorithm (MICA)," J. King Saud Univ. - Eng. Sci., vol. 31, no. 3, pp. 253-261, (2019).

43. A. Yazdandoost, P. Khazaei, R. Kamali, and S. Saadatian, "An Efficient Scheduling for Security Constraint Unit Commitment Problem Via Modified Genetic Algorithm Based on Multicellular Organisms Mechanisms," World Autom. Congr. Proc., vol. 2, pp. 58-63, (2018).

44. X. Fang, L. Bai, F. Li, and B. M. Hodge, "Hybrid component and configuration model for combinedcycle units in unit commitment problem," J. Mod. Power Syst. Clean Energy, vol. 6, no. 6, pp. 13321337, (2018).

45. C. Zhu, F. Lu, H. Zhang, and C. C. Mi, "Robust predictive battery thermal management strategy for connected and automated hybrid electric vehicles based on thermoelectric parameter uncertainty," IEEE J. Emerg. Sel. Top. Power Electron., vol. 6, no. 4, pp. 1796-1805, (2018).

46. S. Maghsudlu and S. Mohammadi, "Optimal scheduled unit commitment considering suitable power of electric vehicle and photovoltaic uncertainty," J. Renew. Sustain. Energy, vol. 10, no. 4, (2018).

47. X. Chen, M. B. Mcelroy, Q. Wu, Y. Shu, and Y. Xue, "Transition towards higher penetration of renewables: an overview of interlinked technical, environmental and socio-economic challenges," J. Mod. Power Syst. Clean Energy, vol. 7, no. 1, (2019).

48. T. K. Renuka, P. Reji, and S. Sreedharan, "An enhanced particle swarm optimization algorithm for improving the renewable energy penetration and small signal stability in power system," Renewables Wind. Water, Sol., vol. 5, no. 1, (2018).

49. T. Ghose, H. W. Pandey, and K. R. Gadham, "Risk assessment of microgrid aggregators considering demand response and uncertain renewable energy sources," J. Mod. Power Syst. Clean Energy, vol. 
7, no. 6, pp. 1619-1631, (2019).

50. M. Bayati, M. Abedi, G. B. Gharehpetian, and M. Farahmandrad, "Short-term interaction between electric vehicles and microgrid in decentralized vehicle-to-grid control methods," Prot. Control Mod. Power Syst., vol. 4, no. 1, (2019).

51. J. B. Mogo and I. Kamwa, "Improved deterministic reserve allocation method for multiarea unit scheduling and dispatch under wind uncertainty," J. Mod. Power Syst. Clean Energy, vol. 7, no. 5, pp. 1142-1154, (2019). 